\title{
La leucoplasia oral. Su implicación como lesión precancerosa
}

\author{
Martínez-Sahuquillo Márquez A*, Gallardo Castillo I**, Cobos Fuentes MJ***, \\ Caballero Aguilar J**, Bullón Fernández P****
}

\section{RESUMEN}

La leucoplasia es definida actualmente en base a un concepto esencialmente clínico. En este sentido, se propone un diagnóstico provisional de leucoplasia basado en criterios clínicos y un diagnóstico definitivo para el que se requiere la eliminación de los factores etiológicos y, ante lesiones persistentes, el examen histopatológico. La consideración de la leucoplasia como lesión precancerosa proviene esencialmente de los estudios de seguimiento en los que se constata su transformación maligna. Pese a que el diagnóstico de displasia es esencialmente subjetivo y a la existencia de algún trabajo reciente que no encuentra relación entre ésta y la evolución posterior de las leucoplasias, la presencia de displasia epitelial en el examen histológico se considera actualmente como el indicador más importante de una mayor probabilidad de transformación maligna de la leucoplasia. Entre otros factores relacionados con un peor pronóstico se consideran el que se trate de una leucoplasia idiopática, las formas clínicas no homogéneas, la localización en cara ventrolateral de la lengua y suelo de boca y un tamaño mayor de 1 centímetro.

Palabras clave: Leucoplasia, precáncer oral, lesión precancerosa, displasia epitelial, pronóstico.

\section{SUMMARY}

The current definition of leukoplakia is based on an essentially clinical concept. Therefore, both a provisional diagnosis of leukoplakia based on clinical criteria and a definite diagnosis for which etiological factors need to be eliminated and, should there be persistent lesions, a histological examination is required, are accepted. Follow up studies in which a malignant transformation has been observed have resulted in leukoplakia being considered as a precancerous disorder. Despite the fact that the diagnosis of dysplasia is subjective and certain recent studies have not found any association between this and the development of leukoplakia, the presence of epithelial dysplasia in the histological examination is currently considered to be the most important indicator of a higher possibility of malignant transformation of the leukoplakia. Among other factors that may indicate a worse prognosis are: if the leukoplakia is idiopathic, if clinical forms are non-homogenous, if it is located on the ventrolateral side of the tongue and the floor of the mouth, and if it is over $1 \mathrm{~cm}$. (0.40 inches) in size.

Key words: Leukoplakia, oral pre-cancer, precancerous lesion, epithelial dysplasia, prognosis.

Fecha de recepción: Enero 2008.

Aceptado para publicación: Enero 2008.

* Profesor Titular de Medicina Bucal. Facultad de Odontología de Sevilla.

** Profesor Asociado de Medicina Bucal. Facultad de Odontología de Sevilla.

*** Colaborador Clínico de Medicina Bucal. Facultad de Odontología de Sevilla.

**** Catedrático de Periodoncia y Medicina Bucal. Facultad de Odontología de Sevilla.

Martínez-Sahuquillo Márquez A, Gallardo Castillo I, Cobos Fuentes MJ, Caballero Aguilar J, Bullón Fernández P. La leucoplasia oral. Su implicación como lesión precancerosa. Av. Odontoestomatol 2008; 24 (1): 33-44. 


\section{CONCEPTO DE LEUCOPLASIA}

Desde el punto de vista etimológico el término leucoplasia significa crecimiento o proliferación blanca. Fue propuesto por primera vez por Schwimmer en 1877 y se utilizó con un sentido anatomoclínico para distinguir a una lesión blanca de las mucosas, principalmente de la bucal, con un destacado pleomorfismo clínico, histológico y etiológico y que en una pequeña proporción de casos podía experimentar una transformación maligna. En 1949, siguiendo a Bernier, se impone un criterio estrictamente histológico al restringir el término de leucoplasia a aquellas lesiones que microscópicamente presentaran disqueratosis, término que se utilizó en contraposición al de displasia (Borrello, 1971).
Es a partir de 1967, cuando la OMS siguiendo a Pindborg, establece el sentido puramente clínico que actualmente se considera inseparable del término leucoplasia. Considera como tal a una "placa blanca que no puede desprenderse por raspado y que no puede clasificarse como ninguna otra lesión" y añade la aclaración de que "no es una entidad histopatológica, porque en ella puede observarse una gran variedad de alteraciones microscópicas". La esencia de dicho concepto ha venido manteniéndose en las distintas reuniones internacionales de consenso celebradas (Tabla I).

En la actualidad, se propone un diagnóstico provisional de leucoplasia basado en criterios clínicos y un diagnóstico definitivo para el que se requiere la eliminación de los factores etiológicos y, ante lesiones persistentes, el examen histopatológico.

\section{TABLA 1.- DEFINICIONES DE LEUCOPLASIA EN LAS REUNIONES DE CONCENSO Y ÚLTIMAS PUBLICACIONES}

\begin{tabular}{|c|c|}
\hline Reuniones de Consenso & Definición de leucoplasia \\
\hline WHO, 1978 & $\begin{array}{l}\text { Una mancha o placa blanca que no puede ser eliminada por el } \\
\text { raspado ni caracterizada clínica o histológicamente como ningu- } \\
\text { na otra enfermedad. }\end{array}$ \\
\hline $\begin{array}{l}1^{\text {a }} \text { Conferencia Internacional sobre } \\
\text { Leucoplasias. Malmo (Suecia). } \\
\text { Axell et al. } 1984\end{array}$ & $\begin{array}{l}\text { Una mancha o placa blanca que no puede ser caracterizada clínica } \\
\text { o histológicamente como ninguna otra enfermedad y que no está } \\
\text { asociada a ningún agente causal físico o químico a excepción de } \\
\text { tabaco. }\end{array}$ \\
\hline $\begin{array}{l}\text { Symposium Internacional de } \\
\text { Lesiones orales blancas. } \\
\text { Uppsala (Suecia). Axell et al. } 1996\end{array}$ & $\begin{array}{l}\text { Una lesión predominante blanca de la mucosa oral que no puede } \\
\text { ser caracterizada como ninguna otra lesión definible, alguna de las } \\
\text { leucoplasias orales puede transformarse en cáncer. }\end{array}$ \\
\hline WHO, 1997. Pindborg et al. & $\begin{array}{l}\text { Una lesión predominante blanca de la mucosa oral que no puede } \\
\text { ser caracterizada como ninguna otra lesión definible. }\end{array}$ \\
\hline $\begin{array}{l}\text { WHO, Pathology } \& \text { Genetics. } \\
\text { Head and neck tumours. } \\
\text { Barnes et al. } 2005\end{array}$ & Ninguna definición. \\
\hline Warnakulasuriya et al. 2007 & $\begin{array}{l}\text { Placas blancas de riesgo cuestionable habiendo excluido (otras) } \\
\text { enfermedades conocidas o alteraciones que no suponen un au- } \\
\text { mento del riesgo de cáncer. }\end{array}$ \\
\hline
\end{tabular}




\section{LA LEUCOPLASIA ORAL COMO LESIÓN PRECANCEROSA}

La OMS ratificó en su última reunión de consenso de 1997 (Pingborg et al 1997) el seguir considerando dentro del precáncer oral a las lesiones precancerosas y a los estados precancerosos. Se considera como lesión precancerosa a "un tejido de morfología alterada más propenso a cancerizarse que el tejido equivalente de apariencia normal" y como estado precanceroso a "una condición generalizada que se asocia con un riesgo significativamente mayor de cáncer". La leucoplasia y la eritroplasia, conjuntamente con la queilitis actínica y la queratosis del paladar asociada al fumar invertido se consideran lesiones precancerosas.

En dos publicaciones recientes, se confirma a la leucoplasia como parte del precáncer oral incluyéndola uno, conjuntamente con la eritroplasia, dentro de las lesiones epiteliales precursoras (Barnes et al, 2005) y el otro en lo que denomina como alteraciones potencialmente malignas (Warnakulasuriya et al, 2007).

La evidencia de que la leucoplasia es una lesión precancerosa proviene principalmente de los estudios que han evidenciado que:

a) Numerosas lesiones de cáncer oral están asociadas a lesiones de leucoplasia cuando son diagnosticadas. b) Una proporción de leucoplasias muestran rasgos morfológicos y citológicos similares a los de COCE (carcinoma oral de células escamosas), salvo invasión.

c) Fundamentalmente, que el seguimiento de algunas leucoplasias ha constatado su transformación maligna.

Los estudios de seguimiento han encontrado un porcentaje de malignización de la leucoplasia que oscila entre el 1 y el $18 \%$. Tales discrepancias podrían obedecer al empleo de criterios diagnósticos no estandarizados y a las diferencias en la selección de los pacientes. En referencia a este último aspecto, los estudios epidemiológicos en la población general muestran cifras menores que los realizados con poblaciones seleccionadas de pacientes (Reibel, 2003) (Tabla 2).

Por otra parte, diversas características etiológicas, clínicas, topográficas e histológicas se han relacionado con el potencial de transformación maligna de las leucoplasias. Otros factores considerados de riesgo serían: el mayor tiempo de evolución de las lesiones y la edad avanzada de los pacientes; el sexo femenino; el tamaño de las lesiones superior a 1 centímetro; la perpetuación de hábitos tóxicos, en particular el tabaco y el alcohol; y la existencia de un estado precanceroso (liquen plano oral, elastosis actínica, etc) (Bermejo, 1998).

TABLA 2.- ESTUDIOS DE SEGUIMENTO SOBRE LA MALIGNIZACIÓN DE LEUCOPLASIAS. MODIFICADO DE REIBEL J ET AL, 2003

\begin{tabular}{|l|c|c|c|}
\hline & $N^{\circ}$ de pacientes & Seguimiento/años & \% malignización \\
\hline Pindborg et al, 1968 & 248 & 3,9 & 4,4 \\
Silverman and Rosen, 1968 & 117 & $1-11$ & 6,0 \\
Kramer et al, 1970 & 187 & $?$ & 4,8 \\
Mehta et al, 1972 & 117 & 10 & 0,9 \\
Silverman et al, 1976 & 4.762 & 2 & 0,13 \\
Bánóczy, 1977 & 670 & 9,8 & 6,0 \\
Silverman et al, 1984 & 257 & 7,2 & 17,5 \\
Lind, 1987 & 157 & 9,3 & 8,9 \\
Schepman et al, 1998 & 166 & 2,5 & 12,0 \\
Holmstrup et al, 2006* & 169 & 6,6 & 4,0 \\
\hline
\end{tabular}

* Estudio retrospectivo. 


\section{CARACTERÍSTICAS ETIOLÓGICAS}

Los factores etiológicos relacionados con la aparición de las leucoplasias coinciden en líneas generales con los implicados en la génesis del precáncer y cáncer oral. No obstante, en un porcentaje importante de los casos no es posible identificar ninguno de ellos, siendo consideradas como leucoplasias idiopáticas.

\section{A) Tabaco}

Actualmente se admite que el tabaco es el factor más claramente relacionado con la producción de leucoplasias, considerándose que alrededor del $80 \%$ se asocian al hábito de fumar. Además, el riesgo de leucoplasia aparece relacionado con la cantidad que se fuma al día y con el tiempo que se lleva fumando.

En referencia a otras formas de consumo de tabaco, estudios realizados en deportistas y otros grupos, relacionan el consumo de diferentes formas de rapé y de tabaco masticable ("tabaco sin humo" en la literatura anglosajona) con una mayor prevalencia de leucoplasia oral (Roed-Petersen et al, 1973). Trabajos realizados en USA han demostrado un vínculo positivo entre el tabaco sin humo y el cáncer de encía, vestíbulo bucal y mucosa yugal (Win et al, 1981; Wisnieswski and Barolucci, 1989). Sin embargo, estudios realizados en Suecia no encuentran esta correlación, atribuyéndolo a una diferente composición del tabaco empleado, sobre todo en lo referente a la concentración de N-nitrosaminas (Larson et al, 1991).

Otras formas de consumo, como el fumar con la candela para adentro, usual en comunidades aisladas de la India, el Caribe y Sudamérica, se vincula con una elevada incidencia de leucoplasia y cáncer en el paladar. En este tipo de consumo hay que considerar el efecto térmico como un cofactor importante.

Por otra parte, un porcentaje considerable de leucoplasias asociadas al tabaco regresan cuando se abandona el hábito. Roed-Petersen y Pindborg, en un estudio en 168 fumadores con leucoplasia encontraron que entre los que disminuían el nivel de consumo a menos de la mitad durante 3 meses en un $50 \%$ mejoraba la lesión y en un 6,2\% desaparecía, mientras que entre los que dejaron de fumar la lesión desaparecía en un 37\% a los 3 meses y en un 58,2\% a los 6 meses (Roed-Petersen et al, 1982). En este mismo sentido, otros autores confirman que alrededor del $80 \%$ de las leucoplasias asociadas al hábito de fumar regresan cuando se abandona el hábito (Gupta et al, 1995; Bánóczy et al, 2001).

También se ha constatado en estudios con un largo seguimiento, un menor riesgo de transformación maligna en las leucoplasias asociadas al tabaco en comparación a las leucoplasias idiopáticas (Silverman et al, 1984, Schepman et al, 1998).

Como características clínicas de las leucoplasias asociadas al tabaco se ha señalado la aparición de finas estrías blanquecinas en su superficie a modo de una "huella digital" (Pinborg, 1980) y una mayor frecuencia de aparición en suelo de boca (Schepman et al, 2001)

\section{B) Infección por Candida}

Entre un $7 \%$ y un $50 \%$ de las lesiones leucoplásicas están infectadas por Candida, especialmente Candida albicans. Si bien en la mayoría de los casos se considera que se tratan de sobreinfecciones de leucoplasias preexistentes, existen datos que apoyan que la infección por Candida pueda representar el principal agente etiológico de algunos tipos de leucoplasia. Así, alrededor de un $10 \%$ de las leucoplasias orales cumplen los criterios clínicos e histológicos descritos por Cawson y Lehner para la leucoplasia candidiásica (candidiasis hiperplásica crónica). De una u otra forma, la invasión por Candidas de las capas queratinizadas de la superficie epitelial de las leucoplasias, particularmente en las no homogéneas, se asocia con una mayor prevalencia de displasia (Renstrup, 1970; Barret 1998). El potencial de malignización de la leucoplasia candidiásica, puede ser explicado en parte por la capacidad que tienen las especies de candidas para catalizar la formación de nitrosaminas carcinogénicas (Krogh et al, 1987a, b).

\section{C) Otros factores}

Las queratosis friccionales son lesiones blancas que aparecen en áreas que son comúnmente traumati- 
zadas, tales como el borde lateral de la lengua, la mucosa bucal a nivel del plano de oclusión y crestas edéntulas y se relacionan de forma evidente con la acción irritativa repetida de agentes traumáticos. Su exclusión dentro del concepto de leucoplasia se realizó basándose en la desaparición de las lesiones al eliminar el factor causal. Sin embargo, la experiencia clínica nos demuestra que es frecuente que lesiones blancas no regresen a pesar de suprimir los posibles agentes traumáticos desencadenantes, sobre todo cuando se trata de lesiones bien establecidas y con un prolongado tiempo de evolución. En estos casos de persistencia de la lesiones debemos considerarlas como leucoplasias. En este sentido, en un estudio clinicopatológico de 110 leucoplasias no encuentran diferencias significativas en lo referente a edad, extensión de las lesiones, formas clínicas y presencia o no de displasia, entre las leucoplasias idiopáticas, las asociadas al tabaco y las relacionadas con factores traumáticos (Bagán y cols. 1993)

La posible implicación del virus del papiloma humano (HPV) en la etiología y en el potencial de malignización de las lesiones precancerosas ha sido extensamente estudiado, no obteniéndose resultados concluyentes. La mayor frecuencia de aislamiento de HPV en lesiones precancerosas y de cáncer que en la mucosa normal y su asociación con los tipos $16 / 18$, justifican investigaciones futuras (Miller and Johnstone, 2001).

Ciertos estados generales se han relacionado con una mayor prevalencia de leucoplasias. En este sentido, diversas situaciones sistémicas en las que se produce atrofia de la mucosa oral, como las deficiencias de vitamina B12 y ácido fólico, la anemia sideropénica y otras carencias nutricionales, determinarían una mayor susceptibilidad a los factores etiológicos locales. Enfermedades tradicionalmente asociadas a la leucoplasia y al cáncer, como la glositis intersticial en la sífilis terciaria y la disfagia sideropénica, han experimentado un notable retroceso en su incidencia.

Los estudios epidemiológicos relacionan el consumo excesivo de alcohol con un aumento del riesgo de cáncer oral y éste se incrementa considerablemente cuando se asocia con el tabaco. Pese a ello, el alcohol raramente es mencionado en los estudios sobre la leucoplasia, exceptuando algún trabajo aislado en el que se la relaciona con un mayor consumo del mismo.

\section{CARACTERÍSTICAS CLÍNICAS}

Las leucoplasias pueden aparecer como una lesión única, localizada, como lesiones difusas, ocupando extensas áreas de la mucosa oral, o incluso como lesiones múltiples. De igual forma, el aspecto clínico de las leucoplasias es muy heterogéneo, pudiendo variar desde áreas maculares, lisas y ligeramente blanquecinas y translúcidas, a placas netamente blancas, elevadas, gruesas, firmes y con superficie rugosa y fisurada. Generalmente son asintomáticas, pudiendo en algunos casos presentar una ligera sensación de ardor.

El característico pleomorfismo de las lesiones leucoplásicas ha dado lugar a la aparición sucesiva de distintas clasificaciones clínicas, en un intento de caracterizar las lesiones y servir de orientación sobre su posible comportamiento. En la actualidad se consideran dos formas clínicas: las leucoplasias homogéneas y las leucoplasias no homogéneas (Axell et al, 1984). Las formas homogéneas son mucho más prevalentes que las no homogéneas, en una proporción que varía en los estudios de $2: 1$ a $11: 1$.

Las leucoplasias homogéneas son definidas como lesiones predominantemente blancas, uniformes, de apariencia delgada y de superficie lisa y suave, a veces cuarteada, arrugada u ondulada. Generalmente sin infección por cándidas y asintomáticas, por lo que suelen descubrirse de forma casual durante una exploración rutinaria.

Las leucoplasias no homogéneas muestran una superficie irregular. Ocasionalmente son sintomáticas y provocan ardor. Dentro de éstas se incluyen la eritroleucoplasia, la leucoplasia nodular y la leucoplasia exofítica.

La eritroleucoplasia es una lesión blanca en la que alternan zonas rojas. La leucoplasia nodular es una lesión blanca con gránulos o nódulos ligeramente 
elevados, redondeados, blanquecinos y/o rojizos. Estos dos tipos se corresponden con la forma clínica descrita por Pindborg como leucoplasia moteada ("speckled" leukoplakia), asociándose con una gran frecuencia a una infección por Candidas (Pindborg et al, 1963). Su importancia viene dada por la frecuencia en que el estudio histológico revela signos de displasia epitelial.

La leucoplasia no homogénea exofítica, anteriormente denominada verrugosa, se caracteriza por aparecer como una lesión blanca con proyecciones irregulares filiformes.

Se ha constatado un riesgo 4-7 veces mayor de transformación maligna en las leucoplasias no homogéneas en comparación con las del tipo homogéneo (Pindborg et al, 1968; Gupta et al, 1989; Silverman et al, 1984; Schepman et al, 1998). Sin embargo, hay que poner de manifiesto como en las leucoplasias homogéneas la tasa de malignización está alrededor de un $5 \%$ en estudios sobre poblaciones de pacientes seleccionados y de un $0,6 \%$ en estudios en población general (Gupta et al, 1989).

Es importante recalcar, que salvo una mayor frecuencia de displasia en las leucoplasias no homogéneas, no existe una correspondencia entre el aspecto clínico y los hallazgos histopatológicos. Esto queda demostrado por trabajos como el de Waldron y Shafer (1975), en el que el análisis histopatológico de 3.256 leucoplasias orales reveló como lesiones de aspecto benigno a veces correspondían a carcinomas infiltrantes y otras leucoplasias con un aspecto clínico de carcinoma sólo mostraban distintos grados de hiperqueratosis (Waldron and Shafer, 1975).

La leucoplasia verrucosa proliferativa, descrita por Hansen, es un tipo muy agresivo de lesión no homogénea, multifocal o difusa y que de forma casi constante evoluciona desarrollando un COCE. En la actualidad hay publicados unos 150 casos, con una mayor incidencia en mujeres que en hombres en una proporción de 4:1, tanto fumadores como no fumadores y una edad media de 60 años (Van der Wall and Reichardt, 2007). El diagnóstico es retrospectivo una vez visto su comportamiento (Bagán et al, 1998).

\section{CARACTERÍSTICAS TOPOGRÁFICAS}

La localización más frecuente de la leucoplasia es la mucosa retrocomisural y luego la mucosa yugal. Otras localizaciones frecuentes son el paladar duro, los rebordes alveolares desdentados, sobre todo en la zona molar de los inferiores, la lengua y el labio, particularmente el inferior. La encía, el paladar blando, la cara ventral de la lengua y el suelo de la boca son localizaciones menos frecuentes. En un porcentaje importante de casos pueden ser bilaterales.

Se han considerado como zonas de alto riesgo al suelo de la boca, la cara ventrolateral de la lengua y el paladar blando, al ser las áreas de mayor incidencia para el COCE y con una mayor exposición a los agentes carcinogénicos. En este sentido, los estudios han señalado un mayor potencial de malignización en las leucoplasias localizadas en estas áreas (Kramer, 1978). Sin embargo, otros estudios han relacionado a las leucoplasias localizadas en el suelo de la boca con el tabaco y no han encontrado una mayor tasa de transformación (Schepman et al 1998). Igualmente, en un estudio reciente, se encontró que el potencial de malignización fue independiente de la localización de la leucoplasia (Holmstrup et al, 2006).

\section{CARACTERÍSTICAS HISTOPATOLÓGICAS}

Los cambios histopatológicos de las leucoplasias son muy diversos, pudiendo variar desde una hiperqueratosis sin displasia hasta diversos grados de displasia epitelial. Las leucoplasias sin displasia epitelial suponen el $80-90 \%$ de todas las leucoplasias. El rasgo histopatológico más constante viene dado por la presencia de hiperqueratosis. Ésta puede ser una ortoqueratosis o una paraqueratosis o incluso estar presente ambas formas de queratinización. En la mayoría de los casos se acompaña de una hiperplasia epitelial debida a un aumento en espesor del estrato espinoso (acantosis) y es frecuente la papilomatosis. En las leucoplasias sin displasia el infiltrado inflamatorio del corion suele ser escaso o estar ausente. Las leucoplasias con displasia epitelial, son menos frecuentes, representando el 10-20\% de todas las leucoplasias. En éstas es más frecuente la 
paraqueratosis y la presencia de un infiltrado inflamatorio a nivel del corion.

La displasia epitelial (literalmente: crecimiento anormal) representa una combinación de las alteraciones celulares individuales (atipias) y de la estructuración normal del epitelio observadas en la transición gradual hacia la malignidad (premalignidad). Entre las alteraciones celulares estarían las siguientes: aumento del tamaño de los nucléolos; hipercromatismo nuclear, pleomorfismo nuclear, aumento de la relación núcleo/citoplasma, aumento de la actividad mitótica, figuras mitóticas anormales y pleomorfismo celular. Los signos displásicos en relación a una desestructuración del epitelio serían: crestas epiteliales en forma de gota, hiperplasia de la basal, hipercelularidad y un patrón alterado en la maduración de los queratinocitos (estratificación epitelial anormal, presencia de mitosis en estratos superficiales, pérdida de adhesión intercelular, queratinización de células del estrato espinoso, etc).

El grado de intensidad de la displasia depende de la frecuencia de los cambios displásicos y de la afectación de los estratos profundos de forma aislada, de los estratos profundos e intermedios o de la totalidad del epitelio. Así, de forma subjetiva, se clasifica la displasia como leve, moderada o severa. Cuando los signos displásicos involucran todo el espesor del epitelio estaremos ante un carcinoma "in situ" (neoplasia intraepitelial), lo que excluye al diagnóstico de leucoplasia. Esta lesión ya no se considera reversible, pudiendo transcurrir varios años hasta que se produce la invasión. Cuando focos de células epiteliales sobrepasan la basal, invadiendo la lámina propia, ya se considera un carcinoma invasivo.

La displasia epitelial se diagnostica con mayor frecuencia en leucoplasias no homogéneas pero también es frecuente en las homogéneas (Shafer and Waldrom 1961, Pindborg et al 1968 and 1977, Mehta et al 1969, Bánózy and Csiba 1976, Silverman 1984, Shepman et al 1998, Lee et al 2000, Cowan et al 2001).

Pese a que el diagnóstico de displasia es esencialmente subjetivo y a la existencia de algún trabajo reciente que no encuentra relación entre esta y la evolución posterior de las leucoplasias, la presencia de displasia epitelial en el examen histológico se considera actualmente como el indicador más importante de una mayor probabilidad de transformación maligna de la leucoplasia. Las leucoplasias con displasia malignizan con una mayor frecuencia que las leucoplasias sin displasia, pero hay que señalar como también malignizan lesiones que no mostraron displasia. Mincer y colaboradores, comprobaron como en un periodo de seguimiento de 8 años de lesiones orales con displasia, el $20 \%$ de éstas evolucionaron a un carcinoma y el $20 \%$ experimentaron un aumento en la severidad de la displasia, mientras que en el $40 \%$ de las lesiones permanecieron sin cambios y el $20 \%$ restante remitieron (Mincer et al, 1972). En un estudio similar, Gupta y colaboradores, encontraron como en un periodo de seguimiento de 10 años la malignización se produjo en menos del $10 \%$ de las lesiones orales displásicas; asimismo, el 30\% empeoraron, el 13\% regresaron y el $50 \%$ permanecieron sin cambios (Gupta et al, 1980).

\section{DIAGNÓSTICO}

El diagnóstico provisional se basa en el estudio clínico de la lesión. Los criterios clínicos incluyen la apariencia, consistencia al tacto y localización de la lesión. Por inspección podremos clasificarla como homogénea o incluirla en alguna subdivisión de las no homogéneas. La palpación meticulosa de la lesión deberá descartar la presencia de induración o dolor a la compresión de los tejidos, lo que estaría más en consonancia con una lesión maligna.

Dentro de la valoración clínica es importante identificar los posibles factores causales, pudiendo catalogar la leucoplasia como idiopática, asociada al tabaco o relacionada con un agente traumático. En este último caso, si en un plazo de 2 a 4 semanas tras la eliminación del agente irritativo no desaparece la lesión se considerará el diagnóstico clínico de leucoplasia.

Finalmente, es preciso realizar un hemograma y una bioquímica sanguínea para detectar posibles estados sistémicos asociados, principalmente una anemia sideropénica. En algunos casos puede estar indicado el practicar una serología luetica. 
El diagnóstico definitivo se lleva a cabo, tras la eliminación de los factores etiológicos, mediante el estudio histopatológico de las lesiones persistentes. Se realizará en todos los casos, incluso cuando se trate de leucoplasias homogéneas con todos los rasgos de benignidad. Su principal objetivo reside en excluir a otras entidades, fundamentalmente el carcinoma de células escamosas. Además de la confirmación del diagnóstico de leucoplasia es importante conocer si existen signos de displasia y el grado de la misma.

En el caso de lesiones pequeñas se recomienda una biopsia escisional, con eliminación completa de la lesión. Cuando se trata de lesiones extensas se realizará una biopsia incisional, incluyendo en la toma el margen lesional y algo del tejido normal adyacente. Deben elegirse las zonas clínicamente sospechosas de malignización, como las áreas eritroplásicas, engrosadas o ulceradas. Si existe más de un área sospechosa, se tomaran varias muestras en la misma sesión. La tinción con azul de toluidina, que muestra apetencia por las áreas displásicas, tiene una baja especificidad, pero puede ser útil como ayuda al elegir la zona a biopsiar.

\section{DIAGNÓSTICO DIFERENCIAL}

Principalmente debe de realizarse con el carcinoma de células escamosas. Si bien, algunos datos clínicos, como la induración y aparición de sintomatología, pueden resultar orientativos, su diferenciación requiere el estudio histopatológico de la lesión.

En el diagnóstico diferencial de la leucoplasia se incluyen aquellos cuadros que cursan con lesiones blancas de la mucosa oral de tipo queratósicas que no pueden ser desprendidas por raspado. Ya nos hemos referido a la queratosis friccional y a la candidiasis hiperplásica crónica. De la misma forma, habría que considerar a los siguientes:

- Leucoedema. Se considera una variación de la normalidad, no precisando tratamiento. Ha sido descrito con mayor frecuencia en la raza negra y relacionado con el hábito de fumar. Aparece como una película difusa, blanco-grisácea, de superficie arrugada, que se extiende por la mu- cosa bucal. Histológicamente se caracteriza por un marcado edema de las células del estrato espinoso, pudiendo haber distintos grados de hiperparaqueratosis. A diferencia de la leucoplasia, que suele mostrar una coloración blanca más definida, el leucoedema se hace menos visible o desaparece al tensar los tejidos.

- Leucoplasia vellosa. Se caracteriza por una lesión blanca de superficie arrugada, no desprendible por raspado, localizada preferentemente en el borde de la lengua de forma bilateral. Su diagnóstico requiere la demostración de la presencia del virus de Epstein-Barr en la lesión. En su defecto, la falta de respuesta al tratamiento con antifúngicos y la demostración de un estado de inmunodeficiencia, se consideran un apoyo importante al diagnóstico de presunción realizado en base a la clínica

- Liquen plano oral. Cuando se presenta como una placa blanca solitaria es bastante difícil de diferenciar de la leucoplasia. De todas formas, es frecuente que los bordes de la placa de LPO tengan unos límites difuminados e incluso aparezca, aunque sea de forma escasa, el típico patrón reticular. Cuando existen lesiones cutáneas el diagnóstico se simplifica. El estudio microscópico es definitivo. Hay casos en los que coexisten las dos entidades.

- Lesiones liquenoides orales (LLO) relacionadas con restauraciones de amalgama de plata. Pueden aparecer en la encía, lengua o mucosa bucal, en la proximidad de distintas restauraciones metálicas, bien como una lesión queratósica en placa como una leucoplasia o bien simulando un liquen plano. En una proporción de los casos, la remoción de las restauraciones metálicas determinará su regresión.

- Lupus eritematoso. Las lesiones discoides orales son frecuentes en los individuos con lesiones cutáneas de lupus discoide y en los que padecen lupus eritematoso sistémico, pudiendo en ocasiones ser la primera manifestación de dichos cuadros. Algunas lesiones discoides orales pueden ser muy semejantes a la leucoplasia y al LPO, si bien suelen tener un mayor componente eritematoso y presentan una característica disposición radial de capilares en la periferia de la placa. Cuando existen, las manifestaciones cutáneas y generales son fundamentales en el diagnóstico. 
- Nevus esponjoso blanco. Es una entidad rara, hereditaria autosómica dominante. Son lesiones blancas, de superficie arrugada, que afectan a varias mucosas. Pueden existir al nacer o hacerse patentes en la pubertad. A nivel oral suele afectar a la mucosa bucal de forma simétrica. Microscópicamente el epitelio está engrosado con marcada espongiosis, acantosis y paraqueratosis. Son asintomáticas, no existiendo evidencia de una posible transformación maligna.

\section{TRATAMIENTO}

El primer paso a considerar en el tratamiento de la leucoplasia, es la supresión de los posibles factores relacionados con su etiología: excluir el tabaco y el alcohol, eliminar cualquier factor irritativo de tipo mecánico, etc.

Es importante recordar la frecuente asociación, bien como agente primario o bien con carácter oportunista, de una infección por Candidas. Es más común en leucoplasias no homogéneas, sobre todo de localización retrocomisural, pero también puede darse en las de tipo homogéneo . Estaría indicado la administración de un tratamiento con antifúngicos tópicos o incluso sistémicos y volver a examinar la lesión a las 2 semanas, siendo frecuente constatar una mejoría muy evidente de la misma. Muchos clínicos realizan dicho tratamiento de forma sistemática en todas las lesiones blancas durante las 2 semanas anteriores a la toma de la biopsia.

Antes de instaurar cualquier tipo de tratamiento es necesario haber realizado un estudio histopatológico de la lesión, para descartar la existencia de malignidad y determinar si hay displasia y el grado de la misma.

La situación más deseable sería la extirpación quirúrgica de todas aquellas leucoplasias que persisten tras la eliminación de los posibles factores etiológicos. Esto es particularmente factible en leucoplasias únicas de pequeño tamaño, cuyo tratamiento se limitaría a la realización de una biopsia escisional, requiriendo posteriormente revisiones periódicas semestrales.
Sin embargo, en lesiones mayores la actitud terapéutica vendría dictada por distintos factores, como el aspecto clínico y localización de la leucoplasia y fundamentalmente, por la histología de la lesión. En general, habría que tratar siempre las leucoplasias no homogéneas, las leucoplasias localizadas en áreas de alto riesgo como el suelo de la boca y cara ventral de la lengua y muy especialmente las leucoplasias con displasia, sobre todo de grado moderado o severo.

En lesiones grandes sin displasia, en las que la cirugía resulta complicada, muchos clínicos aconsejan una actitud expectante: además de asegurar la eliminación de los posibles factores etiológicos, revisar al paciente cada 6 meses y si se produce un cambio de aspecto de la lesión o se vuelve sintomática, volverla a biopsiar. Por el contrario, otros prefieren una actitud intervencionista, eliminando la lesión si en la primera revisión a los 6 meses no muestra una mejoría evidente.

La extirpación de la lesión puede ser llevada a cabo con cirugía convencional, criocirugía o con láser de dióxido de carbono $\left(\mathrm{CO}_{2}\right)$. En las lesiones con displasia estaría especialmente indicada la extirpación quirúrgica con bisturí convencional, ya que permite el análisis histológico posterior de la pieza quirúrgica y una valoración de los bordes de la resección. Con frecuencia requiere la reparación plástica de la zona tratada con colgajos o injertos. La vaporización de la lesión con láser de $\mathrm{CO}_{2}$ generalmente se reserva para leucoplasias extensas sin displasias.

El tratamiento médico con antioxidantes puede ser apropiado en los casos de leucoplasias extensas o en pacientes con alteraciones sistémicas que implican un elevado riesgo quirúrgico. Muchos autores han utilizado la vitamina A por vía sistémica en grandes dosis. Actualmente se utilizan derivados más potentes y menos tóxicos, como el ácido 13 cis-retinoico (isotretionina) y el etretinato a dosis de 1-1,5 $\mathrm{mg} / \mathrm{Kg} /$ día, repartido en tres tomas, durante 3-4 meses, lográndose en un porcentaje importante de los casos una reducción o desaparición de la lesión. Sus principales desventajas derivan de la elevada frecuencia de efectos indeseables (queilitis, eritema facial, descamación cutánea, prurito, alopecia, conjuntivitis, etc.) y la alta tasa de recurrencia de la lesión tras la suspensión del tratamiento. Además, son teratóge- 
nos y tóxicos, por lo que durante su administración debe de evitarse la gestación y controlar en sangre los enzimas hepáticos, colesterol, triglicéridos y glucosa.

Otros antioxidantes, como los betacarotenos (provitamina A) y el alfa-tocoferol (vitamina E) no muestran efectos tóxicos y han demostrado una eficacia similar, con tasas de remisión parcial o total de un 40-60\%. La recidiva se produce casi en todos los casos tras la finalización del tratamiento.

También se ha ensayado la terapia citotóxica tópica, mediante la aplicación de pincelaciones con una solución de sulfato de bleomicina al $1 \%$ en dimetilsulfóxido, durante 5 minutos diariamente hasta cumplir 2 semanas. Estaría indicado en leucoplasias multifocales o localizadas en zonas complejas para la cirugía.

Los pacientes deben de ser revisados periódicamente cada 6-12 meses, aún en los casos en lo que el tratamiento parece haber dado resultado, sobre todo si no hemos logrado controlar de forma adecuada los factores de riesgo.

\section{BIBLIOGRAFÍA}

1. Axell T, Holmstrup P, Kramer IRH, Pindborg JJ, Shear M. International seminar on oral leukoplakia nand associated lessions related to tobacco habits. Comunity Dent Oral Epidemiol. 1984; 12:145-54.

2. Axell T, Pindborg JJ, Smith CJ, Van der Waal I, and an International Collaborative Group on Oral White Lesions, Oral white lesions with special reference to precancerous and tobaco-related lesions: conclusions of an international symposium held in Uppsala. Sweden, May 1821, 1994. J Oral Pathol Med. 1996; 25: 49-54.

3. Bagán JV, Vera F, Milian $M^{a} A$, Peñarrocha $M$, Silvestre FJ, Sanchís JM. Leucoplasia oral: estudio clínico patológico de 110 casos. Arch Odontoestomatol. 1993. 9:127-38.

4. Bagán JV, Lloria E, Jiménez Y, Pérez A. Leucoplasia verrucosa proliferativa. presentación de siete casos. Rev Eur Odonto-Estomatol. 1998; 10:363-70.

5. Bánóczy J, Csiba A. Occurrence of epithelial dysplasia in oral leukoplakia. Analysis and followup study of 12 cases. Oral Surg Oral Med Oral Pathol Oral Radiol Endod 1976; 42:766-74.

6. Bánóczy J, Gintner Z, Dombi C (2001). Tobacco use and oral leukoplakia. J Dent Educ 65:322-7.

7. Barnes L, Eveson JW, Reichardt P, Sidransky D. WHO Clasification of tumours. Pathology and genetics. Head and neck tumours. IARCPress. Lyon, 2005.

8. Barrett AW, Kingsmill VJ, Speight PM. The frequency of fangal infection in biopsies of oral mucosal lesions. Oral Dis 1998; 4:26-31.

9. Bermejo A, López Jornet P. Condición precancerosa y lesión precancerosa. En: Bermejo A. Medicina Bucal. Vol. I. Enfermedades mucocutáneas y de las glándulas salivales. Madrid. Ed. Sintex. 1998: 247-58.

10. Borrello, E. Las lesiones blancas de la mucosa oral. Ed. La Médica. Rosario. 1971.

11. Cowan CG, Gregg TA, Napier SS, McKenna SM, Kee F. Potentially malignant oral lesions in northern Ireland: a 20-year population-based perspective of malignant transformation. Oral Dis 2001; 7:18-24.

12. Gupta PC, Metha FS, Daftari DK, et al, Incidence of oral cancer and natural history of oral precancerous lesions in a 10-year follow-up study of Indian villagers. Comunity Dent Oral Epidemiol. 1980; 8:287-333.

13. Gupta PC, Bhonsle RB, Murti PR, Daftary DK, Mehta FS, Pindborg JJ. An epidemiologic assessment of cancer risk in oral precancerous lesions in India with special reference to nodular leukoplakia. Cancer 1989; 63:2247-52.

14. Gupta PC, Murti PR, Bhonsle RB, Mehta FS, Pindborg JJ. Effect of cessation of tobacco use 
on the incidence of oral mucosal lesions in a 10yr follow-up study of 12,212 users. Oral Dis 1995; 1:54-8.

15. Holmstrup P, Vedtofte P, Reibel J, Stoltze K. Long term tratment outcome of oral premalignant lesions. Oral Oncology 2006 42, 461-74.

16. Krogh P, Hald B, Holmstrup P. Possible mycological etiology of oral mucosal cancer: catalytic potential of infecting Candida albicans and other yeasts in production of Nnitrosobenzylmethylamine. Carcinogenesis 1987a; 8:1543-8.

17. Krogh P, Holmstrup P, Thorn JJ, Vedtofte P, Pindborg JJ. Yeast species and biotypes associated with oral leukoplakia and lichen planus. Oral Surg Oral Med Oral Pathol Oral Radiol Endod 1987b; 63:48-54.

18. Larson $\AA$, Axell T, Andersson G. Reversibility of snuff dipper's lesion in Swedish moist snuff users: a clinical and histologic follow-up study. $J$ Oral Pathol Med. 1991; 20:258-64.

19. Lee JJ, Hong WK, Hittelman WN, Mao L, Lotan $\mathrm{R}$, Shin DM, et al, Predicting cancer development in oral leukoplakia: ten years of translational research. Clin Cancer Res 2000; 6:1702-10.

20. Mehta FS, Pindborg JJ, Gupta PC, Daftary DK. Epidemiologic and histologic study of oral cancer and leukoplakia among 50,915 villagers in India. Cancer 1969; 24:832-49.

21. Miller CS, Johnstone BM. Human papillomavirus as a risk factor for oral squamous cell carcinoma: a meta-analysis, 1982-1997. Oral Surg Oral Med Oral Pathol Oral Radiol Endod 2001; 91:622-35.

22. Mincer HH, Coleman SA, Hopkins KP. Observations on the clinical characteristics of oral lesions showing histologic epitelial dysplasia. Oral Surg Oral Med Oral Pathol. 1972; 33:389-99.

23. Pindborg JJ, Jolst O, Renstrup G, RoedPetersen B. Studies in oral leukoplakia: a preliminary report on the period prevalence of malignant transformation in leukoplakia based on a followup study of 248 patients. J Am Dent Assoc 1968; 76:767-71.

24. Pindborg JJ, Daftary DK, Mehta FS. A follow-up study of sixty-one oral dysplastic precancerous lesions in Indian villagers. Oral Surg Oral Med Oral Pathol Oral Radiol Endod 1977; 43: 38390.

25. Pindborg JJ, Reibel J, Roed-Peterson B, Mehta FS. Tobacco induced changes in oral leukoplakic epithelium. Cancer 1980; 45:2330-36.

26. Pindborg JJ, Reichart P, Smith CJ, van der Waal I. World Health Organization: histological typing of cancer and precancer of the oral mucosa. Berlin: Springer-Verlag. 1997.

27. Reibel, J. Prognosis of oral premalignant lesions: significance of clinical, histopatological and molecular biological caracteristics. Crit Rev Oral Biol Med 2003; 14(1):47-62.

28. Renstrup G. Occurrence of candida in oral leukoplakias. Acta Pathol Microbiol Scand [B] Microbiol Immunol 1970; 78:421-4.

29. Roed-Petersen B, Gupta PC, Pindborg JJ, et al, Assotiation between oral leukoplakias and sex, age and tobacco habits. Bull WHO 1972; 43: 13-9.

30. Roed-Petersen B, Pindborg JJ. A study of Danish snuff-induced oral leucoplakias. J Oral Phatol 1973; 2:301-13.

31. Roed-Petersen B. Effect on oral leukoplakia of reducing or ceasing tobacco smoking. Acta Derm Venereol 1982; 62:164-7.

32. Reibel, J. Prognosis of oral premalignant lesions: significance of clinical, histopatological and molecular biological caracteristics. Crit Rev Oral Biol Med. 2003; 14(1):47-62.

33. Schepman KP, van der Meij EH, Smeele LE, van der Waal I. Malignant transformation of oral 
leukoplakia: a follow-up study of a hospital-based population of 166 patients with oral leukoplakia from The Netherlands. Oral Oncol 1998; 34: 270-5.

34. Schepman KP, Bezemer PD, van der Meij EH, Smeele LE, van der Waal I. Tobacco usage in relation to the anatomical site of oral leukoplakia. Oral Dis 2001; 7:25-7.

35. Shafer WG, Waldron CA. A clinical and histopathologic study of oral leukoplakia. Surg Gynecol Obstet 1961; 112:411-20.

36. Silverman S, Rosen RD. Observations of the clinical characteristics and natural history of oral leukoplakia. J Am Dent Assoc 1968;76:772-7.

37. Silverman S Jr, Gorsky M, Lozada F. Oral leukoplakia and malignant transformation. A follow-up study of 257 patients. Cancer 1984; 53:563-8.

38. Speight PM, Morgan PR. The natural history and pathology of oral cancer and precancer. Community Dent Health. 1993; 10, suppl.1:31-4.

39. an der Waal I, Banoczy J, Axell T, et al, Diagnostic and therapeutic problems of oral precancerous lesions. Int J Oral Maxillofac Surg 1986; 15:790-8.

40. Van der Waal I. Diagnóstico y tratamiento de lesiones precancerosas. FDI World 1995; 2:6-9.

41. Van der Waal I, Reichart PA. Oral proliferative verrucous leukoplakia revisited.Oral Oncol. 2007 Nov 29 [Epub ahead of print].
42. Warnakulasuriya S, Newell W, Jonson I, van der Vaal. Nomenclature and classificatio of potenciallly malingnat disorders of the oral mucosa. J Oral Pathol Med 2007 (in press).

43. Waldrom CA, Shafer WG. Leucoplakia revisited. A clinicopa-thologic study 3256 oral leukoplakias. Cancer. 1975; 36:1386-92.

44. Walker DM, Arendorf TM. Candidadl leucoplakia, chronic multifocal candidiosis and median rhomboid glossitis. En: Samaranayake LP, Mac Farlane TW. Oral Candidiosis. London. Ed. Wright. 1990.

45. Win, DM.; Blot, WJ.; Shy, CM.; Pickle, LW.; Toledo, MA.; Fraumeni, JF. Snuff dipping and oral cancer among women in southern Unites States. New Engl J Med. 1981; 304:745-9.

46. Wisnieswski JF, Barolucci AA. Comparative pattens of smokeless tobacco usage among major league baseball players. J Oral Pathol Med. 1989; 18:322.

47. WHO Colaborating Centre for Oral Precancerous Lesions. Definitions of leukoplakia and related lesions: An aid to s tudies on oral precancer. Oral Surg Oral Med Oral Pathol. 1978; 46:518-39.

\section{CORRESPONDENCIA}

A. Martínez-Sahuquillo Márquez Facultad de Odontología de Sevilla.

Avicena, s/n

41009 Sevilla 